\title{
(2) OPEN ACCESS \\ Skeletal muscle mass in relation to 10 year cardiovascular disease incidence among middle aged and older adults: the ATTICA study
}

\author{
Stefanos Tyrovolas, ${ }^{1,2,3}$ Demosthenes Panagiotakos 주 , 3,4 Ekavi Georgousopoulou, 3,4,5 \\ Christina Chrysohoou, ${ }^{6}$ Dimitrios Tousoulis, ${ }^{6}$ Josep Maria Haro, ${ }^{1,2,7}$ Christos Pitsavos $^{6}$
}

\begin{abstract}
${ }^{1}$ Parc Sanitari Sant Joan de Déu, Fundació Sant Joan de Déu, Dr Antoni Pujades, 42, 08830, Sant Boi de Llobregat, Barcelona, Spain

${ }^{2}$ Instituto de Salud Carlos III, Centro de Investigación Biomédica en Red de Salud Mental, CIBERSAM, Monforte de Lemos 3-5. Pabellón 11, 28029, Madrid, Spain

${ }^{3}$ Department of Science of Dietetics and Nutrition, School of Health Science and Education, Harokopio University, Athens, Greece

${ }^{4}$ Department of Nutrition and Dietetics, Faculty of Health, University of Canberra, Canberra, Australian Capital Territory, Australia

${ }^{5}$ Medical School, The Australian National University, Canberra, Australian Capital Territory, Australia

${ }^{6}$ First Cardiology Clinic, School of Medicine, University of Athens, Athens, Greece ${ }^{7}$ Department of Medicine, Universidad de Barcelona, Barcelona, Spain
\end{abstract}

\section{Correspondence to} Dr Stefanos Tyrovolas, Parc Sanitari Sant Joan de Déu, Fundació Sant Joan de Déu, CIBERSAM,Dr. Antoni Pujadas, 42, 08830 - Sant Boi de Llobregat, Barcelona, Spain; s.tyrovolas@pssjd.org

Received 12 February 2019 Revised 6 August 2019 Accepted 16 September 2019 Published Online First 11 November 2019

\section{Check for updates}

(C) Author(s) (or their employer(s)) 2020. Re-use permitted under CC BY-NC. No commercial re-use. See rights and permissions. Published by BMJ.

To cite: Tyrovolas $\mathrm{S}$,

Panagiotakos $\mathrm{D}$

Georgousopoulou E, et al. J

Epidemiol Community Health 2020;74:26-31.

\section{ABSTRACT}

Background Skeletal muscle mass (SMM) is inversely associated with cardiometabolic health and the ageing process. The aim of the present work was to evaluate the relation between SMM and 10 year cardiovascular disease (CVD) incidence, among CVD-free adults 45+ years old.

Methods ATTICA is a prospective, population-based study that recruited 3042 adults without pre-existing CVD from the Greek general population (Caucasians; age $\geq 18$ years; 1514 men). The 10 year study follow-up (2011-2012) captured the fatal/non-fatal CVD incidence in 2020 participants (50\% men). The working sample consisted of 1019 participants, 45+ years old (men: $n=534$; women: $n=485$ ). A skeletal muscle mass index (SMI) was created to reflect SMM, using appendicular skeletal muscle mass (ASM) standardised by body mass index (BMI). ASM and SMI were calculated with specific indirect population formulas.

Results The 10 year CVD incidence increased significantly across the baseline SMI tertiles $(p<0.001)$. Baseline SMM showed a significant inverse association with the 10 year CVD incidence (HR 0.06, 95\% Cl 0.005 to 0.78 ), even after adjusting for various confounders. Additionally, participants in the highest SMM tertile had $81 \%$ (95\% Cl 0.04 to 0.85 ) lower risk for a CVD event as compared with those in the lowest SMM tertile. Conclusions The presented findings support the importance of SMM evaluation in the prediction of long-term CVD risk among adults 45+ years old without pre-existing CVD. Preservation of SMM may contribute to CVD health.

\section{INTRODUCTION}

The global population is ageing at an unprecedented speed, especially in Europe. As a concept, ageing is considered a continuous process starting from birth and is accompanied by various physiological changes and a number of comorbidities ${ }^{12}$ that affect health and quality of life. ${ }^{34}$ Skeletal muscle mass $(\mathrm{SMM})^{4}$ alterations are among these physiological changes.

SMM tissue decline, as a part of these physiological changes, starts in middle age (or even earlier, in the 30s) and progresses in more advanced age. It has been shown that SMM declines with a rate of more than $3 \%$ per decade starting from the age of $30+{ }^{6}$ Half of the human body's mass is actually SMM and it has an active role in numerous metabolic pathways. ${ }^{7}$ SMM decline is related, among others, to various disability patterns, poor mental health and increased mortality. ${ }^{58}$ In addition, well documented studies have shown that SMM alterations are related to cardiovascular health, ${ }^{9} 10$ even different muscle morphology. ${ }^{11}$ Recently, Srikanthan et $a l^{12}$ reported the importance of muscle tissue in relation to cardiovascular disease (CVD) and total mortality in stable CVD patients.

It is well known that CVD is the major cause of mortality and morbidity in middle aged and older populations; specifically, in Greece, among those aged 50 to 70 years CVDs were related to more than 7295 disability-adjusted life years (DALYs) per 100000 in 2017 (https://vizhub.healthdata. org/gbd-compare/). Moreover, although Greece is considered to be a country with a high life expectancy, recent studies report an increase in the incidence of non-fatal CVD. ${ }^{13}$ Although various classical factors, such as unhealthy nutrition, excess body weight, smoking, physical inactivity, psychological disorders and low socioeconomic status, have long been considered among the major determinants for CVD development in middle aged people, ${ }^{13}$ the causal CVD pathway is still complicated. Moreover, due to the population's increased longevity, new factors have arisen (eg, muscle tissue loss, multimorbidity, etc), making CVD prevention even more complex.

Greece has a CVD risk profile that is similar to the other Mediterranean countries (Spain, France, Italy, Portugal, etc) and, despite the pronounced CVD and musculoskeletal disorders epidemic in these areas, ${ }^{13} 14$ there are no epidemiological data on SMM and CVD risk. To the best of our knowledge, there have been limited studies evaluating the effect of SMM on CVD risk among only patients with stable $\mathrm{CVD},{ }^{12}$ but none among CVD-free populations. Given the complexity of the CVD "web of causation" implicating classical and new factors, the association of SMM with cardiometabolic health and the ageing process, and the lack of data among Mediterranean (and other European) CVD-free populations, the aim of the present work was to evaluate the relation between SMM and 10 year CVD incidence in the ATTICA study cohort among adults $45+$ years old without previous CVD. Since evidence is emerging that targeted intervention programmes can prevent muscle tissue alterations, this study could provide useful information for effective, non-pharmacological CVD health promotion and planning. 


\section{METHODS}

\section{Study sample}

In brief, ATTICA is a prospective, population-based, cohort study performed in the Athens metropolitan region of Attica (Greece). The study recruited 3042 non-institutionalised adults (Caucasians; women/men: 1528/1514; $18+$ yearsold) without previous CVD, out of a total of 4053 individuals who initially asked to participate (no differences as regards age and gender were observed between the individuals who asked to participate and those finally

recruited). Of the 3042 participants, 1544 (50.8\%) were $45+$ yearsold. The enrolment of the participants was carried out during 2001-2002, and after that two follow-up waves followed 5 (in 2006) and 10 years (in 2012) later. Random, multistage sampling based on the age and gender distribution of the reference population, as defined by the Hellenic National Statistical Service Census Survey of 2001, was applied. Sampling procedures anticipated enrolling only one participant per household, while institutionalised individuals were excluded from participating in the study. All participants underwent detailed baseline assessments which included medical history, physical examination and blood sampling for biochemical measurements. Baseline CVD was excluded in all participants by the study physicians. The ATTICA study was approved by our Institutional Ethics Committee (ID001/2002) and conformed to the ethical guidelines of the 1975 Declaration of Helsinki, with all participants providing written informed consent. The ATTICA methodology is described in detail elsewhere. ${ }^{15}$

\section{Baseline study assessments}

\section{Sociodemographic, bioclinical and lifestyle measurements}

Comprehensive baseline data were collected, including demographic details (eg, age, gender and years of education), personal/family history of cardiometabolic disease (eg, hypertension, diabetes, hypercholesterolaemia and CVD), and information about dietary and other lifestyle habits (eg, physical activity and smoking in pack years), as described previously. ${ }^{15}$ Briefly, all participants underwent a detailed baseline dietary evaluation through the EPIC-Greek questionnaire, ${ }^{16}$ which is a validated semi-quantitative food-frequency questionnaire that was kindly provided by the Unit of Nutrition of the Athens Medical School. Moreover, the MedDietScore was used to assess the adherence to the Mediterranean diet. ${ }^{17}$ Physical activity levels of each participant were also assessed at baseline using the International Physical Activity Questionnaire (IPAQ). ${ }^{18}$

At baseline, morning (8-10 am) fasting blood samples were obtained from all participants after overnight fasting (10-12 hours without food/alcohol intake, except for water). Triglyceride, total cholesterol and high-density lipoprotein cholesterol levels were measured by a standard chromatographic enzymatic method. Low-density lipoprotein cholesterol was calculated using the Friedewald formula. ${ }^{19}$ Hypercholesterolaemia was defined as total cholesterol $>200 \mathrm{mg} / \mathrm{dL}$ or treatment with lipid-lowering drug(s). Moreover, subjects with fasting blood glucose $>125 \mathrm{mg} /$ $\mathrm{dL}$ or on antidiabetic treatment were classified as having diabetes. Selected circulating pro-inflammatory biomarkers were also measured, namely high sensitivity C-reactive protein (CRP), as well as tumour necrosis factor $\alpha(\mathrm{TNF} \alpha)$ and interleukin-6 (IL-6), following the specific protocols. ${ }^{20}$ Furthermore, resting arterial blood pressure (average of three recordings in the sitting position) was also measured at baseline, and individuals exhibiting an average blood pressure $\geq 140 / 90 \mathrm{mmHg}$ or taking antihypertensive medication(s) were categorised as hypertensive.
Skeletal muscle mass definition and calculation

Standardised measurements of anthropometric parameters were performed by trained study researchers at baseline, including body weight and height as well as waist circumference and hip circumference, in order to calculate the body mass index (BMI: body weight in kilograms divided by the height in metres squared; $\mathrm{kg} / \mathrm{m}^{2}$ ). Individuals with BMI values $>29.9 \mathrm{~kg} / \mathrm{m}^{2}$ were grouped as obese. SMM was calculated throughout the appendicular skeletal muscle mass (ASM) only for the Caucasian population, based on the equation proposed by Lee et al. ${ }^{21}$ This indicator was further adjusted by BMI to create a skeletal muscle mass index (SMI) as the proportion of ASM/BMI. ${ }^{22}$ In addition, SMI tertiles were created with the first tertile defining the low muscle mass.

\section{CVD incidence at the 10 year follow-up (2002-2012)}

The 10 year follow-up was conducted during 2011-2012 (median follow-up period: 8.41 years), as previously described. ${ }^{13}$ Briefly, 2583 of the 3042 initial participants were re-assessed during the 10 year follow-up (participation rate $85 \%$ ). No differences were observed for age and gender distributions, as compared with the total sample (baseline age (mean \pm SD): $45 \pm 14$ and $46 \pm 14$ years for women and men, respectively; of these 1370 (53\%) were $45+$ years old). However, of those who participated in the follow-up, accurate data regarding the development of CVD (and exact date) were obtained from 2020 participants; 1019 of them were over 45 years old (with mean age of $56 \pm 9$ years, men $n=534$ and women $n=485$ ), and comprised the working sample of this work. The definition of the investigated outcomes was based on the International Classification of Diseases, 10th version (ICD-10). In particular, information about the health status of the participants concerned development of: (a) myocardial infarction, angina pectoris, other identified forms of ischaemia (ICD-9 coding (or 10th edition) 410-414.9, 427.2, 427.6 (I20-I25)), and coronary revascularisation (414.01) (ie, coronary artery bypass surgery and percutaneous coronary intervention); (b) heart failure of different types (400.0-404.9, 427.0-427.5, 427.9, 428.- (I50.2-)), and chronic arrhythmias (I49.-); (c) development of stroke (430-438 (I63.-)) (ie, cases (a) to (c) defined CVD event); as well as (d) development of hypertension, hypercholesterolaemia and diabetes (see below for diagnosis). As regards individuals who might have first suffered from a stroke and then had coronary heart disease (CHD), it was a priori decided that the first outcome should be considered as the endpoint, but that the consequent event should also be recorded for further testing of competing risks (however, there were no such cases in the sample). For those participants who died during the follow-up, relevant information was retrieved from their relatives and from the death certificates. ${ }^{13}$

\section{Statistical analysis}

Normally distributed continuous variables are presented as mean \pm SD values, and categorical variables as frequencies. Normality was tested using the Shapiro-Wilk criterion; the nonnormally distributed variables are presented as median and first and third tertile. Crude, non-fatal and fatal incidence rates of combined CVD (ie, cases (a) to (c) as described above) were calculated as the ratio of new cases to the number of participants at the 10 year follow-up. Associations between categorical variables were tested by the $\chi^{2}$ test, whereas associations between continuous variables were tested by the Pearson's $r$ or Spearman's $\rho$ coefficients for normally distributed and skewed variables, respectively. Continuous variables were tested for 
Table 1 Cardiovascular disease (CVD) cases during the ATTICA study 10 year follow-up and key baseline characteristics of the participants $45+$ years old when categorised by baseline muscle mass tissue (SMI) tertiles ( $n=1019)$

\begin{tabular}{|c|c|c|c|c|}
\hline & \multicolumn{3}{|c|}{ Baseline SMI (ASM/BMI) tertiles } & \multirow[b]{2}{*}{$P$ value } \\
\hline & $\begin{array}{l}\text { First tertile } \\
(n=340)\end{array}$ & $\begin{array}{l}\text { Second tertile } \\
(n=344)\end{array}$ & $\begin{array}{l}\text { Third tertile } \\
(\mathrm{n}=335)\end{array}$ & \\
\hline CVD during 10 year follow-up, $n$ (\% yes) & $108(31.7 \%)$ & $117(34.0 \%)$ & $47(14.0 \%)^{*}$ & $<0.001$ \\
\hline \multicolumn{5}{|l|}{ Baseline characteristics } \\
\hline Age (years) & $57.1 \pm 9.2$ & $57.3 \pm 9.4$ & $52.2 \pm 6.3^{*}$ & $<0.001$ \\
\hline Gender (\% male) & $7(02.0 \%)$ & $299(87.0 \%)^{*}$ & $335(100.0 \%)^{*}$ & $<0.001$ \\
\hline Smoking (\% yes) & $136(40 \%)$ & $227(66 \%)^{*}$ & $235(70.0 \%)^{*}$ & $<0.001$ \\
\hline Physical activity (\% physically active) & $129(38.0 \%)$ & $138(40.0 \%)$ & $151(45.0 \%)$ & 0.07 \\
\hline MedDietScore (range 0-55) & $24.0 \pm 6.0$ & $22.2 \pm 6.1^{*}$ & $24.1 \pm 4.0$ & $<0.001$ \\
\hline Education (years at school) & $10.1 \pm 4.1$ & $10.2 \pm 4.1$ & $13.3 \pm 4.0^{*}$ & $<0.001$ \\
\hline SES (low/middle vs high) & $58(17.3 \%)$ & $62(18.0 \%)$ & $151(44.5 \%)^{*}$ & $<0.001$ \\
\hline Obesity (\% yes) & $82(23.8 \%)$ & $110(32.5 \%)$ & $34(10.8 \%)^{*}$ & $<0.001$ \\
\hline Hypertension (\% yes) & $139(41.0 \%)$ & $169(49.0 \%)^{*}$ & $134(40.1 \%)$ & 0.007 \\
\hline Diabetes mellitus (\% yes) & $41(12.0 \%)$ & $55(16.0 \%)$ & $27(08.0 \%)^{*}$ & 0.001 \\
\hline Hypercholesterolaemia (\% yes) & $187(55.2 \%)$ & $172(49.6 \%)$ & 184 (52.0\%) & 0.15 \\
\hline
\end{tabular}

Continuous variables are presented as mean $\pm \mathrm{SD}$, and categorical as counts and relative frequencies (percentages). $\mathrm{P}$ values refer to the comparisons using $\chi^{2}$ test or $\mathrm{t}$-test for the categorical and continuous variables, respectively; values for the between SMI group comparisons (vs first tertile) were corrected using the Bonferroni rule.

${ }^{*} \mathrm{p}<0.05$.

ASM, appendicular skeletal muscle mass; BMI, body mass index; MedDietScore, adherence to the Mediterranean diet; SES, socioeconomic status; SMI, skeletal muscle mass index.

normality via P-P plots. For normally distributed variables, comparisons of means between participants who developed CVD and those who remained CVD-free were performed by the Student's t-test, after controlling for equality of variances by the Levene's test. For continuous variables without normal distribution, comparisons were performed by the non-parametric Mann-Whitney U-test. Cox proportional hazards models were used to estimate the hazard ratios (HR) and the corresponding 95\% confidence intervals $(95 \% \mathrm{CI})$ of developing a CVD event during the study follow-up according to the baseline SMM (SMI) adjusted for other characteristics of participants (gender, education, socioeconomic level-model 1; smoking, physical activity, Mediterranean diet adherence-model 2; hypertension, diabetes, hypercholesterolaemia, obesity—model 3). Assumption of proportionality of hazards was graphically assessed; collinearity between covariates was tested through the correlation coefficients of the models' parameters. Model's goodness-of-fit was evaluated using $-2 \operatorname{logLikelihood}$ (the lower the better). All $\mathrm{p}$ values are based on two-sided tests. Statistical analyses were performed using the Statistical Package for Social Sciences version 22 (SPSS Inc, Chicago, IL, USA).

\section{RESULTS}

The fatal or non-fatal 10 year CVD incidence in the cohort was $26.7 \%$. Crude incidence (risk) of CVD, and various sociodemographic, lifestyle and bioclinical characteristics of the $45+$ year old participants, by baseline SMI tertiles, are presented in table 1 . The lowest 10 year CVD incidence was noted in the highest baseline SMI tertile, compared with the lowest and middle ones (all $\mathrm{p}$ values $<0.05$ ). The prevalence of hypertension, diabetes and obesity was lower among the participants of the highest baseline muscle mass tertile (all $\mathrm{p}$ values $<0.007$ ). In addition, participants grouped in this tertile were younger, mostly men, smokers, more physically active, educated, had a high socioeconomic level and were more adherent to the Mediterranean diet (ie, higher MedDietScore) (all p values $<0.07$ ).

Table 2 presents the hazard ratios (HR and corresponding $95 \% \mathrm{CI}$ ) of the survival models assessing the baseline SMI on the 10 year CVD incidence. Sociodemographic variables (gender, education, socioeconomic level-model 1), as well as certain lifestyle factors (smoking, physical activity, Mediterranean diet adherence-model 2) and clinical parameters (hypertension, diabetes, hypercholesterolaemia, obesity-model 3) were entered into the models. The analysis revealed that baseline SMI levels had a consistent and independent inverse association with 10 year CVD incidence (HR 0.06, 95\% CI 0.005 to 0.78 ) among $45+$ year old participants (table 2). Except for SMI, education level and adherence to the Mediterranean diet were also associated with lower 10 year CVD hazard (HR $0.91,95 \%$ CI 0.84 to 0.99 , and HR $0.95,95 \%$ CI 0.91 to 0.99 , respectively), while the presence of hypertension (HR 1.64, 95\% CI 1.05 to 2.57) and male gender (HR 3.7, 95\% CI 1.32 to 10.5 ) were related to higher 10 year CVD hazard among individuals $45+$ yearsold. Men had 6.1 times higher risk of incident CVD than women (95\% CI 2.45 to 15.18). As regards other anthropometric characteristics, increased BMI was associated with higher CVD hazard (HR 1.34, 95\% CI 1.001 to 1.07) after adjusting for the same group of covariates as with SMI, whereas waist circumference (HR 1.002, 95\% CI 0.98 to 1.02) as well as waist-to-hip ratio were not significantly associated with CVD hazard in the studied sample (HR 2.60, 95\% CI 0.32 to 21.19).

Moreover, as expected, SMI values were inversely associated with IL-6 (age, sex adjusted b-coefficient ( $p$ value): $-0.11 \pm 0.01$ $(\mathrm{p} \leq 0.001)), \quad$ CRP $\quad(b$-coefficient $\quad(p$ value $):-0.37 \pm 0.002$ $(\mathrm{p} \leq 0.001))$ and fibrinogen ( $b$-coefficient ( $\mathrm{p}$ value): $-12.54 \pm 3.34$ $(p<0.001))$. However, further mediation analysis showed no influence of the aforementioned inflammatory markers on the SMM-CVD relationship (data not shown here). Moreover, when BMI was entered in the final model replacing SMI, the estimated model showed lower goodness-of-fit as compared with the one which included SMI (ie, higher - 2logLikelihood as compared with model 3).

A variety of previous studies have shown a strong effect between gender and muscle mass loss on human health. ${ }^{126}$ In line with previous studies, a significant interaction between gender and SMI was also observed in the present work ( $\mathrm{p}$ for 
Table 2 Results from the applied Cox proportional hazard models evaluating the 10 year risk of developing a cardiovascular disease event (outcome) according to the baseline values of muscle mass, for the ATTICA participants 45+ years old

\begin{tabular}{|c|c|c|c|}
\hline \multirow[b]{2}{*}{ All participants } & \multicolumn{3}{|c|}{ Hazard ratios (HR)t, 95\% Cls } \\
\hline & Model 1 & Model 2 & Model 3 \\
\hline SMI (ASM/BMI) & $0.03^{* *}(0.003$ to 0.24$)$ & $0.05^{*}(0.005$ to 0.58$)$ & $0.06 *(0.005$ to 0.78$)$ \\
\hline Men vs women & $6.10 * *(2.45$ to 15.18$)$ & $4.10^{* *}(1.57$ to 10.71$)$ & $3.70 *(1.32$ to 10.53$)$ \\
\hline Education (years at school) & $0.92^{*}(0.85$ to 0.99$)$ & $0.92(0.85$ to 1.00$)$ & $0.91 *(0.84$ to 0.99$)$ \\
\hline SES (low/middle vs high) & 1.32 (0.65 to 2.70$)$ & 1.33 (0.65 to 2.72$)$ & $1.55(0.73$ to 3.29$)$ \\
\hline MedDietScore (range 0-55) & - & $0.95^{*}(0.92$ to 0.99$)$ & $0.95^{*}(0.91$ to 0.99$)$ \\
\hline Smoking ever (yes vs no) & - & $1.14(0.73$ to 1.78$)$ & 1.23 (0.77 to 1.98$)$ \\
\hline Physically active (yes vs no) & - & $0.94(0.61$ to 1.43$)$ & 0.93 (0.59 to 1.44$)$ \\
\hline Obesity (yes vs no) & - & - & $0.66(0.38$ to 1.15$)$ \\
\hline Hypertension (yes vs no) & - & - & $1.64^{*}$ (1.05 to 2.6$)$ \\
\hline Diabetes mellitus (yes vs no) & - & - & 1.64 (0.87 to 3.09$)$ \\
\hline Hypercholesterolaemia (yes vs no) & - & - & $1.15(0.73$ to 1.80$)$ \\
\hline
\end{tabular}

${ }^{*} \mathrm{p}<0.05{ }^{* *} \mathrm{p}<0.001$

†Hazard ratios derived from semi-parametric Cox proportional hazards models.

ASM, appendicular skeletal muscle mass; BMI, body mass index; MedDietScore, adherence to the Mediterranean diet; SES, socioeconomic status; SMI, skeletal muscle mass index.

interaction $<0.001)$. Thus, to further test the role of gender, the previous analysis was stratified in males and females. The SMI levels were inversely associated with the 10 year CVD incidence in $45+$ year old males (HR $0.03,95 \%$ CI 0.001 to 0.744 ), but not in females (HR $0.18,95 \%$ CI 0.002 to 17.13 ).

In order to further explore the potential dose-dependent association of SMM with CVD incidence, further analysis was applied by SMI tertiles. Table 3 presents the hazard ratios of the regression model assessing the 10 year CVD risk by baseline SMI tertiles. Based on the applied analysis, only individuals in the highest SMI tertile as compared with those in the lowest had a significantly lower hazard for developing a CVD event (HR $0.19,95 \%$ CI 0.04 to 0.85 ).

\section{DISCUSSION}

Although SMM has an important cardiometabolic effect, ${ }^{1023}$ until now there have been no data from Europe and the Mediterranean

Table 3 Results from Cox proportional hazards model that evaluated the 10 year cardiovascular disease (CVD) risk by baseline muscle mass tertile, in the ATTICA study participants $45+$ years old.

\begin{tabular}{lll}
\hline & HR & $95 \%$ Cl \\
\hline Men vs women & 3.83 & 0.97 to 15.2 \\
\hline Education (years at school) & $0.91^{*}$ & 0.84 to 0.99 \\
\hline SES (low/middle vs high) & 1.67 & 0.78 to 3.56 \\
\hline MedDietScore (range 0-55) & $0.95^{* *}$ & 0.91 to 0.98 \\
\hline Smoking ever (yes vs no) & 1.26 & 0.79 to 2.02 \\
\hline Physically active (yes vs no) & 0.92 & 0.59 to 1.44 \\
\hline Obesity (yes vs no) & 0.66 & 0.38 to 1.14 \\
\hline Hypertension (yes vs no) & $1.69^{*}$ & 1.08 to 2.65 \\
\hline Diabetes mellitus (yes vs no) & 1.55 & 0.82 to 2.94 \\
\hline Hypercholesterolaemia (yes vs no) & 1.16 & 0.74 to 1.83 \\
\hline SMI (ASM/BMI) & & \\
\hline \multicolumn{1}{c}{ First tertile } & Reference & \\
\hline Second tertile & 0.46 & 0.11 to 1.81 \\
\hline Third tertile & $0.19^{*}$ & 0.04 to 0.85 \\
\hline
\end{tabular}

\section{${ }^{*} p<0.05{ }^{* *} p<0.001$.}

ASM, appendicular skeletal muscle mass; BMI, body mass index; MedDietScore, adherence

to the Mediterranean diet; SES, socioeconomic status; SMI, skeletal muscle mass index. basin investigating the role of muscle tissue on CVD risk among CVD-free populations. The present study reports an inverse association between SMM (as reflected by SMI) preservation and the 10 year CVD incidence in a large community-based cohort of CVD-free adults $45+$ years old. Notably, this relation was independent of the dietary habits, socioeconomic level and multiple well-established CVD risk factors such as smoking habits, hypertension, hypercholesterolaemia and diabetes. Further analysis by SMM subgroups (tertiles) shows that only the subgroup of the highest SMM had the lowest 10 year CVD risk. To the best of our knowledge, this is the first study to evaluate the association between SMM and CVD risk among adults without previous CVD. These results point to the importance of SMM preservation in relation to CVD risk and the public health actions that need to be taken to improve CVD health in middle aged and older populations.

SMM has been shown to be related to various health outcomes, not only in older adults but also among middle aged populations. ${ }^{2}$ SMM has been shown to be inversely related to CVD health and outcomes such as cardiometabolic health, insulin secretion and resistance, metabolic syndrome and diabetes. ${ }^{9} 102324$ These data are in line with the applied unadjusted analysis where the high baseline SMM participant's group had a lower prevalence of CVD risk factors such as obesity, diabetes and hypertension. Muscle tissue has been reported to be associated with endocrine declines (ie, testosterone and growth hormone levels) in parallel with the body's ongoing ageing process, explaining the initial interaction analysis between age and SMM. Despite increasing evidence of the correlation between SMM and CVD health among middle aged and older populations, so far only studies involving CVD or CHD patients have reported the association between muscle tissue, obesity, CVD risk and all-cause mortality risk. ${ }^{12} 25$ This has been noted as the 'obesity-lean' paradox, describing the effect of body composition changes among CVD patients, which may not apply in our analysis where the population was initially CVDfree because of the diverse pathophysiology (ie, cardiorespiratory fitness). ${ }^{26} \mathrm{SMM}$ is known for its major role in the glucose pathway in the human body, highlighting its metabolic significance. ${ }^{24}$ Taking into account the well-known association of insulin resistance with $\mathrm{CVD},{ }^{27}$ it is reasonable to conclude that the metabolic function of SMM has an impact on CVD risk. The aforementioned major 
metabolic pathway could possibly explain the reported association between SMM and the 10 year CVD incidence.

To further explore the effect of SMM preservation on 10 year CVD incidence, SMM (as reflected by SMI) tertile analysis was applied. It was considered that only the highest tertile of SMM conferred protection against CVD risk as compared with the lowest tertile of SMM. In addition, inflammatory ${ }^{28} 29$ and metabolic interrelated pathways ${ }^{1}$ could possibly explain the reported association between SMM preservation and 10 year CVD incidence. Specifically, inflammation markers such as TNF $\alpha$, IL-6 and CRP have been inversely related to skeletal muscle tissue, ${ }^{29}{ }^{30}$ as a part of complex physiological pathways. To test the aforementioned, a multi-adjusted regression model between selected biomarkers (IL-6, CRP and fibrinogen) and the SMI was applied (having the same confounders as those in table 3), without altering the already observed association between SMI and CVD incidence.

Furthermore, previous cross-national studies have reported that male gender is related to higher skeletal muscle mass. ${ }^{2}$ Based on that, we also analysed separately males and females and it was revealed that SMM influences the cardiometabolic risk of males but not of females. This association could be partly explained by the different nature of ageing (ie, endocrine, testosterone levels) among genders and its interrelation to the metabolic-CVD pathway. In addition, the 'gender paradox' in healthy ageing (being female is related to a higher comorbidity burden) ${ }^{31}$ could also explain the different gender effect of SMM in CVD health reported in our analysis.

Currently middle aged and older individuals in Europe and the Mediterranean basin are experiencing poor quality of life due to musculoskeletal disorders in parallel with increased CVD risk. ${ }^{14}$ Progressive SMM loss, in addition to causing declines in gait speed and muscle strength, could lead to sarcopenia, even in the 50+ year old population, ${ }^{2}$ a condition related to multimorbidity, disability and increased healthcare expenses. ${ }^{32}$ These associations-combined with the fact that the Greek population, like other populations in the Mediterranean area, is ageing and the CVD burden is increasing raise major concerns about the need for early non-pharmacological measures in order to promote CVD health. Specific nutrition and physical strategies are the factors that could be possible targets for community interventions and health education campaigns in order to avoid the decline in lean mass. Recent studies have shown that health promotion programmes and interventions that include exercise strategies (ie, periodic physical activity, resistance training) and high protein intake under a specific plan ${ }^{33}$ could help to preserve muscle mass in ageing. ${ }^{34} 35$ Stakeholders in Greece could consider the muscle mass decline among the CVD risk factors for the country's middle aged and older population. Muscle mass preservation through resistance exercise and specific dietary habits within the Mediterranean diet could serve as cost-effective actions for CVD prevention in the community of middle aged and older adults. ${ }^{36}$ Of course, future studies should evaluate physical activity, nutritional and other lifestyle interventions to attain a beneficial SMM and potentially decrease long-term CVD risk.

\section{Strengths and limitations}

The present study has major strengths. It is the first study to evaluate the association between SMM (as reflected by SMI), and the 10 year CVD incidence of a large sample of CVD-free adults aged $45+$ years. The use of a CVD-free population is really important, since cardiac patients have a different physiology, different lifestyle habits, functional limitations and disabilities that may mediate the investigated association between SMM and CVD incidence. In addition, this research work shows the role of muscle mass as a potential determinant of CVD health among middle aged and older subjects. This finding could serve as benchmark in the development of CVD prevention programmes in ageing communities.

Among the study's limitations, it should be noted that the baseline/entry study examination was conducted once and could be susceptible to a certain degree of measurement error. However, the applied methodology is similar to well-known longitudinal, CVD-outcome epidemiological studies, and followed standardised and validated protocols/methods regarding the data collection. Moreover, the enrolment of participants only from a specific area, and without any history of chronic disease, may limit the generalisation of the findings to other populations due to selection bias. Additionally, in studies like the present one the correspondence between CVD-specific hazard and cumulative incidence is usually lost. ${ }^{37}$ This has a very important implication; specifically, the way in which covariates are associated with the hazards may not coincide with the way these covariates are associated with the cumulative incidence. Thus, the results should be interpreted with caution. Regarding the current analysis, the CVD rate was not constant across time and, thus, the implication of cumulative incidence could be misleading. The assessment of SMM was based on population equations that may under- or overestimate the actual SMM rates. However, it is well known that these formulas are validated and present good agreement with the classical method of dual-energy X-ray absorptiometry (DXA) measurement. ${ }^{21}{ }^{38}$ Epidemiologic studies in diverse settings have used these formulas (ASM, ASM/BMI) for the calculation of SMM. ${ }^{2}$ Also, despite the fact that assessments of functioning, such as grip strength and gait speed, are related to muscle mass and older adult's health, the ATTICA study did not include such information. Furthermore, not including in the analysis additional measurements of obesity (such as waist circumference or other effects of body fat) may also limit our findings on the kind of association between BMI, waist circumference or body fat and CVD incidence. Finally, the fact that the present analysis was based only on baseline SMI values is an additional limitation, especially for capturing the effect of decline in SMI over time on CVD incidence.

\section{CONCLUSION}

The present work evaluated the role of SMM on CVD incidence in $45+$ year old adults without pre-existing CVD. It is of major interest nowadays to study the SMM transition in order to better understand its role on CVD outcomes. Throughout this study, the importance of SMM preservation to prevent

\section{What is already known on this subject}

Skeletal muscle mass declines with increasing of age. Alterations in skeletal muscle mass are related to various disability patterns, mental diseases, cardiovascular health and mortality.

\section{What this study adds}

- Limited studies have evaluated the effect of skeletal muscle mass on cardiovascular risk only in patients with stable cardiovascular disease, but there have been no studies involving cardiovascular disease-free populations, until now.

- The findings of this study support the importance of skeletal muscle mass evaluation in the prediction of long-term cardiovascular disease risk among middle aged and older adults without pre-existing cardiovascular disease. 
long-term CVD risk among the middle aged/older population has been highlighted. The prevention of SMM decline, which is becoming increasingly prevalent among middle aged and older populations, may constitute an effective means of promoting CVD health.

Acknowledgements The authors would like to thank the ATTICA study group of investigators: Yannis Skoumas, Natasa Katinioti, Labros Papadimitriou, Constantina Masoura, Spiros Vellas, Yannis Lentzas, Manolis Kambaxis, Konstanitna Paliou, Vassiliki Metaxa, Agathi Ntzouvani, Dimitris Mpougatas, Nikolaos Skourlis, Christina Papanikolaou, Aikaterini Kalogeropoulou, Evangelia Pitaraki, Alexandros Laskaris, Mihail Hatzigeorgiou, Athanasios Grekas, and Eleni Kokkou for assistance in the initial physical examination and/or the follow-up evaluation; Efi Tsetsekou for her assistance in psychological evaluation and follow-up evaluation, as well as the laboratory team: Carmen Vassiliadou and George Dedousis (genetic analysis), Marina Toutouza-Giotsa, Constantina Tselika and Sia Poulopoulou (biochemical analysis), and Maria Toutouza for the database management. We would also like to thank all the participants of the ATTICA study.

Contributors Study design: ST and DBP. Data analysis: ST, DBP. Data interpretation: ST, DBP, ENG, CC, JMH, DT, CC, and CP. Drafting manuscript: ST and DBP. Revising manuscript content: ENG, CC, JMH, DT, CC, and CP. Approving final version of manuscript: All authors.

Funding The ATTICA study is supported by research grants from the Hellenic Cardiology Society (HCS2002) and the Hellenic Atherosclerosis Society (HAS2003). This work was supported by the Ageing Trajectories of Health: Longitudinal Opportunities and Synergies (ATHLOS) project, which has received funding from the European Union's Horizon 2020 research and innovation program under grant agreement No 635316. Stefanos Tyrovolas's work was supported by the Foundation for Education and European Culture (IPEP), the Sara Borrell postdoctoral program (reference no. CD15/00019 from the Instituto de Salud Carlos III (ISCIII - Spain) and the Fondos Europeo de Desarrollo Regional (FEDER).

Competing interests None declared.

Patient consent for publication Not required.

Provenance and peer review Not commissioned; externally peer reviewed.

Data availability statement Data are available upon reasonable request.

Open access This is an open access article distributed in accordance with the Creative Commons Attribution Non Commercial (CC BY-NC 4.0) license, which permits others to distribute, remix, adapt, build upon this work non-commercially, and license their derivative works on different terms, provided the original work is properly cited, appropriate credit is given, any changes made indicated, and the use is non-commercial. See: http://creativecommons.org/licenses/by-nc/4.0/.

\section{ORCID iD}

Demosthenes Panagiotakos http://orcid.org/0000-0001-8583-153X

\section{REFERENCES}

1 Sakuma K, Yamaguchi A. Sarcopenic obesity and endocrinal adaptation with age. Int J Endocrinol 2013;2013:1-12.

2 Tyrovolas S, Koyanagi A, Olaya B, et al. Factors associated with skeletal muscle mass, sarcopenia, and sarcopenic obesity in older adults: a multi-continent study. J Cachexia Sarcopenia Muscle 2016;7:312-21.

3 Landi F, Cruz-Jentoft AJ, Liperoti R, et al. Sarcopenia and mortality risk in frail older persons aged 80 years and older: results from ilSIRENTE study. Age Ageing 2013:42:203-9.

4 Liguori I, Russo G, Aran L, et al. Sarcopenia: assessment of disease burden and strategies to improve outcomes. Clin Interv Aging 2018;13:913-27.

5 Baumgartner RN, Koehler KM, Gallagher D, et al. Epidemiology of sarcopenia among the elderly in New Mexico. Am J Epidemiol 1998:147:755-63.

6 Volpi E, Nazemi R, Fujita S. Muscle tissue changes with aging. Curr Opin Clin Nutr Metab Care 2004;7:405-10.

7 Newman AB, Kupelian V, Visser M, et al. Strength, but not muscle mass, is associated with mortality in the health, aging and body composition study cohort. J Gerontol A Biol Sci Med Sci 2006;61:72-7.

8 Hsu Y-H, Liang C-K, Chou M-Y, et al. Association of cognitive impairment, depressive symptoms and sarcopenia among healthy older men in the Veterans retirement community in southern Taiwan: a cross-sectional study. Geriatr Gerontol Int 2014;14(Suppl 1):102-8.

9 Shishikura K, Tanimoto K, Sakai S, et al. Association between skeletal muscle mass and insulin secretion in patients with type 2 diabetes mellitus. Endocr J 2014;61:281-7.
10 Kim Y, Han B-D, Han K, et al. Optimal cutoffs for low skeletal muscle mass related to cardiovascular risk in adults: the Korea National health and nutrition examination survey 2009-2010. Endocrine 2015;50:424-33.

11 Andersen $\mathrm{K}$, Lind L, Ingelsson E, et al. Skeletal muscle morphology and risk of cardiovascular disease in elderly men. Eur J Prev Cardiol 2015;22:231-9.

12 Srikanthan P, Horwich TB, Tseng CH. Relation of muscle mass and fat mass to cardiovascular disease mortality. Am J Cardiol 2016;117:1355-60.

13 Panagiotakos DB, Georgousopoulou EN, Pitsavos C, et al. Ten-year (2002-2012) cardiovascular disease incidence and all-cause mortality, in urban Greek population: the ATTICA study. Int J Cardiol 2015;180:178-84.

14 Haro JM, Tyrovolas S, Garin N, et al. The burden of disease in Spain: results from the Global Burden of Disease Study 2010. BMC Med 2014;12:236.

15 Pitsavos C, Panagiotakos DB, Chrysohoou C, et al. Epidemiology of cardiovascular risk factors in Greece: aims, design and baseline characteristics of the ATTICA study. BMC Public Health 2003:3:32.

16 Katsouyanni Ket al. Reproducibility and relative validity of an extensive semiquantitative food frequency questionnaire using dietary records and biochemical markers among Greek schoolteachers. Int J Epidemiol 1997;26(90001):118S-27.

17 Panagiotakos DB, Pitsavos C, Stefanadis C. Dietary patterns: a Mediterranean diet score and its relation to clinical and biological markers of cardiovascular disease risk. Nutr Metab Cardiovasc Dis 2006;16:559-68.

18 Papathanasiou G, Georgoudis G, Papandreou M, et al. Reliability measures of the short International Physical Activity Questionnaire (IPAQ) in Greek young adults. Hellenic J Cardiol 2009:50:283-94.

19 Friedewald WT, Levy RI, Fredrickson DS. Estimation of the concentration of low-density lipoprotein cholesterol in plasma, without use of the preparative ultracentrifuge. Clin Chem 1972;18:499-502.

20 Kyrou I, Panagiotakos DB, Kouli G-M, et al. Lipid accumulation product in relation to 10-year cardiovascular disease incidence in Caucasian adults: the ATTICA study. Atherosclerosis 2018;279:10-16.

21 Lee RC, Wang Z, Heo M, et al. Total-body skeletal muscle mass: development and cross-validation of anthropometric prediction models. Am J Clin Nutr 2000:72:796-803.

22 Studenski SA, Peters KW, Alley DE, et al. The FNIH sarcopenia project: rationale, study description, conference recommendations, and final estimates. J Gerontol A Biol Sci Med Sci 2014;69:547-58.

23 Moon JH, Choo SR, Kim JS. Relationship between low muscle mass and metabolic syndrome in elderly people with normal body mass index. J Bone Metab 2015;22:99-106.

24 Srikanthan P, Karlamangla AS. Relative muscle mass is inversely associated with insulin resistance and prediabetes. findings from the Third National Health and Nutrition Examination Survey. J Clin Endocrinol Metab 2011:96:2898-903.

25 Wannamethee SG, Shaper AG, Whincup PH, et al. The obesity paradox in men with coronary heart disease and heart failure: the role of muscle mass and leptin. Int J Cardiol 2014;171:49-55.

26 Lavie CJ, De Schutter A, Patel D, et al. Body composition and coronary heart disease mortality —an obesity or a lean paradox? Mayo Clin Proc 2011;86:857-64.

27 Papakonstantinou E, Lambadiari V, Dimitriadis G, et al. Metabolic syndrome and cardiometabolic risk factors. Curr Vasc Pharmacol 2014;11:858-79.

28 Costamagna D, Costelli P, Sampaolesi M, et al. Role of inflammation in muscle homeostasis and myogenesis. Mediators Inflamm 2015;2015:1-14.

29 Wåhlin-Larsson B, Wilkinson DJ, Strandberg E, et al. Mechanistic links underlying the impact of C-reactive protein on muscle mass in elderly. Cell Physiol Biochem 2017:44:267-78

30 Visser M, Pahor M, Taaffe DR, et al. Relationship of interleukin-6 and tumor necrosis factor-alpha with muscle mass and muscle strength in elderly men and women: the health ABC study. J Gerontol A Biol Sci Med Sci 2002;57:M326-32.

31 Tyrovolas S, Haro JM, Mariolis A, et al. Successful aging, dietary habits and health status of elderly individuals: a k-dimensional approach within the multi-national MEDIS study. Exp Gerontol 2014;60:57-63.

32 Janssen I, Shepard DS, Katzmarzyk PT, et al. The healthcare costs of sarcopenia in the United States. J Am Geriatr Soc 2004:52:80-5.

33 Oikawa SY, Holloway TM, Phillips SM. The impact of step reduction on muscle health in aging: protein and exercise as countermeasures. Front Nutr 2019;6.

34 Freiberger E, Sieber C, Pfeifer K. Physical activity, exercise, and sarcopenia - future challenges. Wien Med Wochenschr 2011:161:416-25.

35 Beavers KM, Ambrosius WT, Rejeski WJ, et al. Effect of exercise type during intentional weight loss on body composition in older adults with obesity. Obesity 2017:25:1823-9.

36 Tyrovolas S, Haro J-M, Mariolis A, et al. Skeletal muscle mass and body fat in relation to successful ageing of older adults: the multi-national MEDIS study. Arch Gerontol Geriatr 2016;66:95-101.

37 Andersen PK, Geskus RB, de Witte T, et al. Competing risks in epidemiology: possibilities and pitfalls. Int J Epidemio/ 2012;41:861-70.

38 Cawthon PM, Peters KW, Shardell MD, et al. Cutpoints for low appendicular lean mass that identify older adults with clinically significant weakness. J Gerontol A Biol Sci Med Sci 2014;69:567-75. 